\title{
CT texture analysis of abdominal lesions - Part I.: Liver lesions
}

IMAGING

\section{REVIEW ARTICLE}

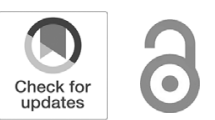

IMAGING 13 (2021) 1, 13-24

DOI: $10.1556 / 1647.2021 .00007$

(c) 2021 The Author(s)

*Corresponding author. Korányi Sándor u. 2, H-1083 Budapest, Hungary.

E-mail: budai.bettina@med. semmelweis-univ.hu

\author{
BETTINA KATALIN BUDAI* $\odot$, VERONICA FRANK $\odot$, \\ SONAZ SHARIATI, BENCE FEJÉR, AMBRUS TÓTH ๑, \\ VINCE ORBÁN, VIKTOR BÉRCZI ๑ and PÁL NOVÁK KAPOSI ๑
}

Department of Radiology, Medical Imaging Centre, Semmelweis University, Faculty of Medicine, Budapest, Hungary

Received: August 6, 2020 • Accepted: April 20, 2021

\begin{abstract}
Artificial Intelligence and the use of radiomics analysis have been of great interest in the last decade in the field of imaging. CT texture analysis (CTTA) is a new and emerging field in radiomics, which seems promising in the assessment and diagnosis of both focal and diffuse liver lesions. The utilization of CTTA has only been receiving great attention recently, especially for response evaluation and prognostication of different oncological diagnoses. Radiomics, combined with machine learning techniques, offers a promising opportunity to accurately detect or differentiate between focal liver lesions based on their unique texture parameters. In this review article, we discuss the unique ability of radiomics in the diagnostics and prognostication of both focal and diffuse liver lesions. We also provide a brief review of radiogenomics and summarize its potential role of in the non-invasive diagnosis of malignant liver tumors.
\end{abstract}

\section{KEYWORDS}

radiomics, texture analysis, machine learning, abdominal imaging, liver

\section{Introduction}

Computed tomography (CT) is a highly effective tool for the detection and characterization of hepatic lesions. Based on the classic morphological signs, CT can be used to diagnose whether a tissue is normal or abnormal visually. However, medical images store and evaluate more detail than what is visible to the trained eyes of specialists.

Texture analysis is a mathematical method, which can quantify the distribution patterns of gray-level intensity and the interrelationships between voxels within a selected region of interest (ROI). It provides a method to measure intra-regional heterogeneity and to detect tissue changes, including subtle differences in textural information that would be imperceivable to the human eye. Radiomics analysis of the liver has been of great interest in the last decade (Fig. 1).

Texture analysis was first applied to medical images by Sutton et al. [1] in 1972, who used the texture analysis of X-ray images for the automated classification of pulmonary diseases, nevertheless the term 'radiomics' first appeared in 2012 in the literature [2]. The definition of radiomics extends beyond texture analysis and includes all high-throughput techniques that can extract a large number of quantitative image features from radiological images. These techniques and the definitions of the radiomic parameters are summarized in the manual of the Image Biomarker Standardization Initiative (IBSI) aimed to standardize feature extraction methods [3].

The process of radiomics-based machine learning analysis can be divided into distinct steps (Fig. 2). In the first step, an ROI is either manually or semi-automatically selected on the acquired standard of care radiographic images (Fig. 3). Radiomic features are then 


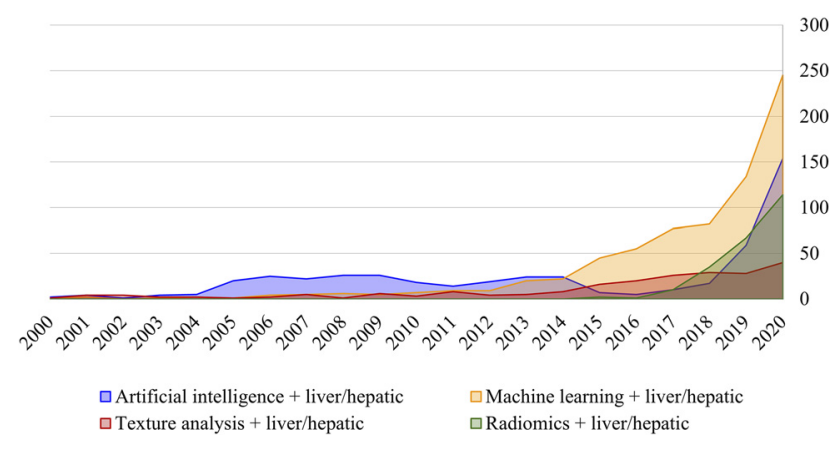

Fig. 1. The increasing number of articles found in PubMed ${ }^{\circledR}$ for keywords Artificial intelligence; Machine learning; Texture analysis; Radiomics published between 2000-2020

extracted from this ROI [4] (Fig. 4). The following step involves feature selection methods. The features are compared with the patient's clinical data, and by using machine algorithms predictive models can be constructed from the selected features [4]. The model is usually trained using internal cross-validation, and the trained model is validated against an independent test set to test its generalizability. The result is a radiomic model, which can be used to guide clinical decision making and provide more personalized medical care for patients in the future.

\section{Non-oncological applications of CTTA}

The number of patients with liver diseases is increasing worldwide. Medical imaging, especially ultrasound and CT, plays an important role in the detection and monitoring of both focal and diffuse liver diseases. In previous studies, texture analysis has been applied to describe both benign and malignant focal changes of the liver. In recent years, CT texture analysis (CTTA) has also gained popularity as a promising novel tool for the non-invasive assessment of diffuse liver diseases. Naganawa et al. [5], who first applied CT texture analysis to detect non-alcoholic steatohepatitis (NASH), reported that CT texture analysis on unenhanced CT scans could predict NASH accurately with an area under the receiver operating characteristic curve (AUC) value of 0.94 in patients without suspected fibrosis. However, the accuracy of their model was markedly lower in patients with liver fibrosis. Other studies showed that CTTA could be used successfully on routinely performed portal venous phase scans for the characterization of incidentally detected liver fibrosis (Table 1). The initial research was conducted on either a single cross-section of the liver $[6,7]$ or on small cuboid regions of interests [8-10]. Three-dimensional texture analysis has only become available recently for describing cirrhosis-related changes in all three dimensions of the liver volume [11]. In a recent study, Budai et al. demonstrated that texture analysis on the entire threedimensional volume of the liver can facilitate the diagnosis of advanced fibrosis and could be a promising tool for identifying high-risk patients. The trained support vector machine and random forest classifiers achieved good to excellent accuracies and their prediction performance seemed to be robust against the type of CT scanners [12].

In a linchpin publication, Lubner et al. evaluated CTTA for the assessment of hepatic fibrosis in 459 patients with chronic hepatitis $\mathrm{C}$ virus [13]. The authors manually drew an ROI around the liver at a single slice of the portal venous phase CT at the level of the porta hepatis. The Couinaud segments I-III and the Couinaud segments IV-VIII were also delineated with a second and a third ROI. Next, the authors extracted first-order radiomic parameters from the ROIs, collected the laboratory parameters, and calculated the APRI and FIB-4 scores for each patient. After dimension reduction, they constructed a multivariable logistic regression model to predict liver fibrosis using mean gray-level intensity, mean of positive pixels, skewness, and kurtosis. Mean gray-level intensity showed the best accuracy with an AUC of 0.81 [95\% confidence interval (CI):0.77-0.85] for F0 vs. $\mathrm{F} 1-4$; $\mathrm{AUC}=0.80$ [95\% CI:0.76-0.83] for F0-1 vs. F2-4; and $\mathrm{AUC}=0.82$ [95\% CI:0.79-0.86] for F0-2 vs. F3-4. The AUC of the multivariable logistic regression model was 0.81 [95\% CI:0.78-0.85] for F0 vs. F1-4, 0.81 [95\% CI:0.78-0.85] for F0-1 vs. F2-4, and 0.86 [95\% CI:0.82-0.89] for F0-2 vs. F3-4 stage fibrosis. The AUC of the APRI score was 0.68 [95\% CI:0.62-0.74], 0.72 [95\% CI:0.67-0.76], and 0.78 [95\% CI:0.74-0.82], and the FIB-4 score was 0.73 [95\% CI:0.680.79], 0.75 [95\% CI:0.70-0.80], and 0.81 [95\% CI:0.77-0.85] in the same comparisons. Based on their results, Lubner et al. highlighted that CTTA did not seem to be as accurate in detecting liver fibrosis as liver surface nodularity or liver segmental volume ratio combined with splenic volume measurement. In a subsequent study, the same research group created a multiparametric model for the staging of liver fibrosis using 11 parameters consisted of radiomic parameters extracted from portal venous phase CT scans and laboratory measures [14]. The model combining all 11 parameters showed AUCs for discriminating significant fibrosis $(\mathrm{ZF} 2)$, advanced fibrosis $(\mathrm{ZF} 3)$, and cirrhosis (F4) of $0.928,0.956$, and 0.972 , respectively. At the same time, a two-parameter model combining liver-surface nodularity with FIB-4 score also showed good accuracy, with AUC values of $0.886,0.915$, and 0.932 in similar comparisons. The above studies demonstrate that CT radiomics analysis combined with laboratory-based biomarkers can improve the diagnostic ability of CT for liver fibrosis [14].

\section{Oncological applications of CTIA}

\section{Radiomics analysis of primary liver tumors}

Radiomics analysis has multiple advantages over visual inspection in the evaluation of circumscribed liver lesions, as it can be used for the objective assessment of tumor heterogeneity at different enhancement phases. Meanwhile, variations in CT protocols and image reconstruction techniques can interfere with the reproducibility of CTTA parameters, which can hinder clinical applications based on CTTA. 


\section{Medical images \\ - Post-processing \\ - Reconstruction \\ - Normalization}

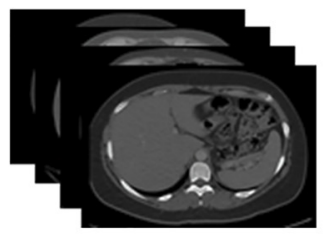

Filtering

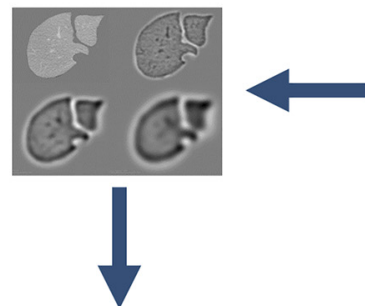

Segmentation

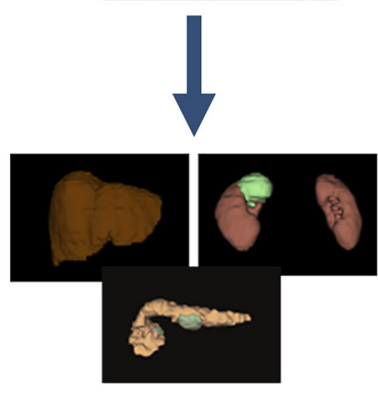

Texture analysis
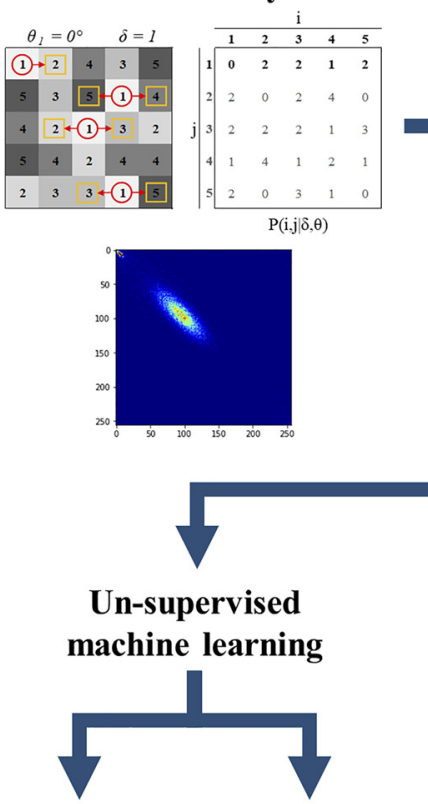

Clustering Dimensionality reduction
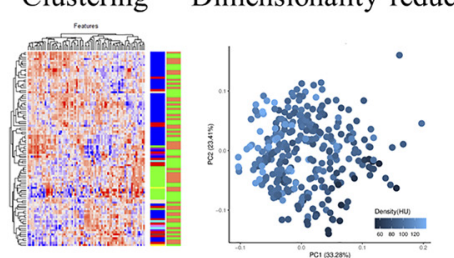

Un-supervised machine learning

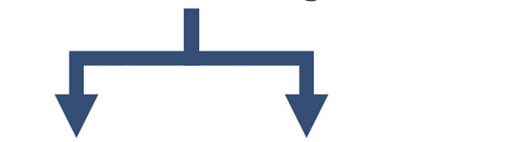

Radiomic data

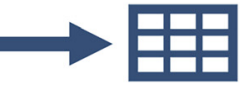

- Transformation

- Discretization

- Normalization

\section{Feature selection methods}

- Recursive feature elimination

- Correlation filtering

- ROC-AUC analysis

- etc.
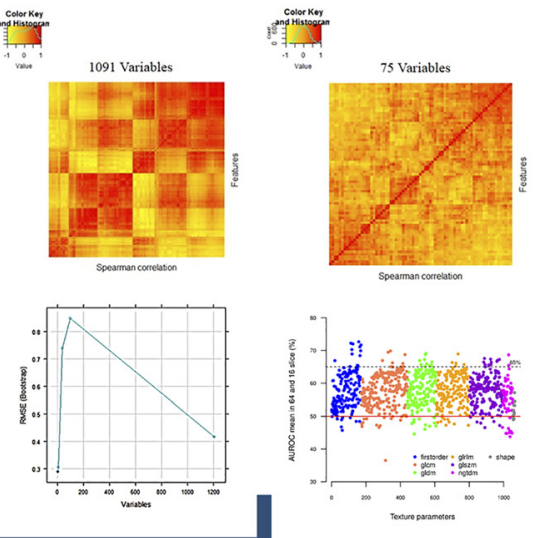

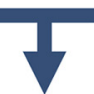

Supervised machine learning

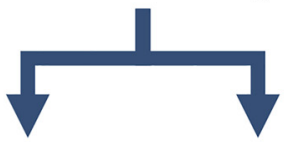

Regression

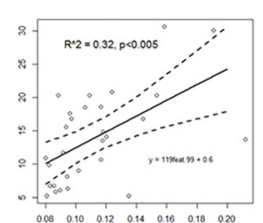

Deep learning

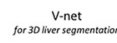

Fig. 2. * Overview diagram of machine learning-based analysis of medical images.

Sequential steps using machine learning algorithms from image acquisition to data results: image post-processing, segmentation of ROI, filtration step, extraction of radiomic data, feature selection, unsupervised or supervised machine learning methods.

ROC-AUC: Area under the receiver operating characteristic curve; ROI: region of interest

${ }^{*}$ Central Illustration

Changes in contrast injection rate and pixel resolution have been shown to weaken the reproducibility of texture parameters derived either from focal liver lesions or the surrounding parenchyma [15]. Elaborated image processing and rigorous data analysis methods are needed to overcome the problems with the reproducibility of the features; however, at the moment, there is no universally accepted system for standardized analysis.

Hepatocellular carcinoma (HCC) is the most common primary liver tumor, and it is one of the leading causes of 


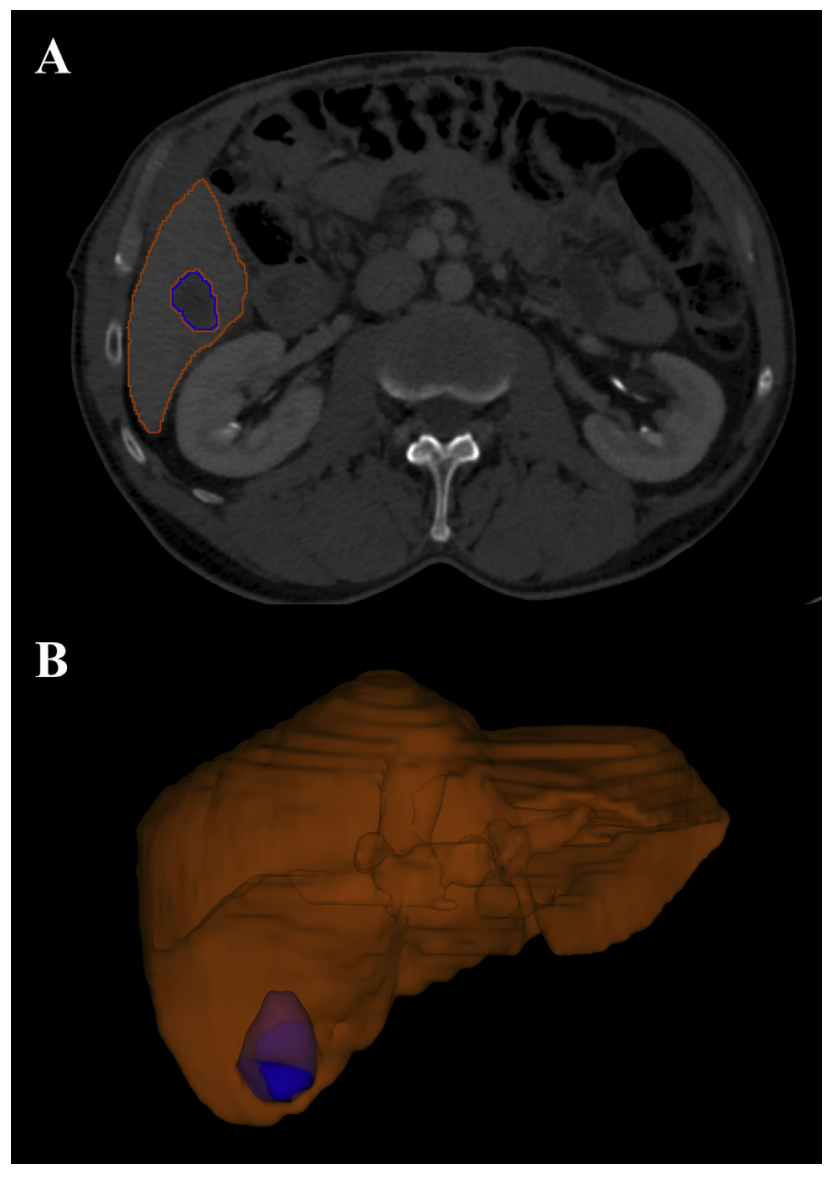

Fig. 3. Manual three-dimensional segmentation of the liver parenchyma and hepatic tumor on portal venous phase axial CT scan. Slice-wise manual segmentation of the area of the whole liver on the axial view of portal venous phase CT scan (A). Three-dimensional reconstruction of the delineated liver tumor and tumor-free parenchyma (B)

cancer-related mortality worldwide [16]. The accurate assessment of the disease status in HCC is essential for establishing the prognosis and deciding between therapeutic options. The Barcelona Clinic Liver Cancer (BCLC) is a well-established staging system of HCC, which integrates data on both tumor extent and the severity of the liver disease [17]. In case there are no signs of significant portal hypertension or severe liver function impairment, surgical resection remains the standard treatment for early-stage HCC. Otherwise, in patients with a chronic liver disease where portal hypertension is also present, liver transplantation is the most appropriate treatment alternative if the tumor stage remains within the Milan criteria [18].

In hepatocellular carcinomas, CTTA was able to identify features that were correlated with tumor grade as well as with the disease-free survival (DFS) of patients after resection (Table 2). A study by Oh et al. [19] demonstrated that the skewness of the pixel intensity histogram at arterial phase CT images is an independent negative predictor of DFS in resected HCC. Skewness greater than 0.3 at filtered images, which proved to have higher predictive power compared to conventional clinicopathologic features such as tumor size or microvascular invasion, could be linked to a higher proportion of brightly enhancing pixels in the arterial phase, and may represent an increased rate of angiogenesis as well as vascular permeability. Other first-order CTTA parameters such as standard deviation and mean of positive pixels showed strong correlation with histology grade and were significantly higher at the arterial phase images of highgrade than at those of low-grade HCC.

Defour et al. [20] reported similar findings. They identified skewness derived from the pixel distribution histogram at portal venous (PV) phase CT images as an independent predictor of overall survival after the surgical resection of HCC. The inter-reader agreement between texture features was weak at the arterial phase and good to excellent at the PV phase. The authors also concluded that CTTA of HCC may assist in selecting the best candidates for resection. Another radiomics-based signature was also able to predict HCC recurrence after liver transplantation. During the analysis of 93 cases in training and 40 cases in the validation set with the least absolute shrinkage and selection operator (LASSO) Cox regression model, Guo et al. [21] identified a subset of predictive features extracted from pre-transplant arterial or PV phase CT scans. They found that the prediction model based on arterial phase features showed better

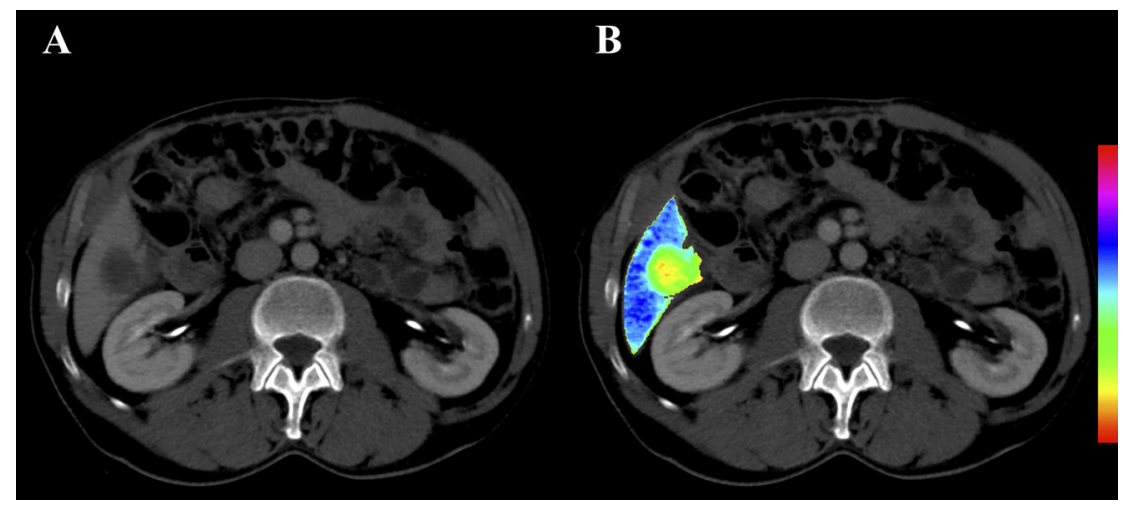

Fig. 4. Result of texture analysis of liver tumor compared with healthy parenchyma on portal venous phase CT scan.

Portal venous phase CT scan (A) and feature map of gray-level run-length matrix-based texture parameter (high gray-level run emphasis) in axial view (B) 
Table 1. Overview of the literature on the CT texture analysis-based staging of liver fibrosis

\begin{tabular}{|c|c|c|c|c|c|}
\hline Study & Description & Segmentation technique & Texture features & Machine learning & Results \\
\hline Daginawala et al. [6] & $\begin{array}{l}\text { Identifying texture features that } \\
\text { shows association with liver } \\
\text { fibrosis stage. }\end{array}$ & $\begin{array}{l}5 \text { consecutive slices at the level } \\
\text { of the porta hepatis }\end{array}$ & $\begin{array}{l}\text { Histogram-based first-order statistics, } \\
\text { GLCM, GLRLM, GLGM-based features, } \\
\text { Laws' features }\end{array}$ & - & $\begin{array}{c}\text { ROC-AUC analysis } \\
\text { F0-2 vs. F3-6: } \\
\text { Median: AUC }=\mathbf{0 . 6 9}[0.57-0.80] \\
\text { F0-3 vs. F4-6: } \\
\text { SRHGE: AUC }=\mathbf{0 . 7 5}[0.62-0.88] \\
\text { F0-4 vs. F5-6: } \\
\text { SRHGE: AUC }=\mathbf{0 . 7 6}[0.64-0.89]\end{array}$ \\
\hline Lubner et al. [7] & $\begin{array}{l}\text { Identifying texture features that } \\
\text { shows association with liver } \\
\text { fibrosis stage. }\end{array}$ & $\begin{array}{l}\text { Single slice of the liver at the } \\
\text { level of the porta hepatis. }\end{array}$ & Histogram-based first-order statistics & - & $\begin{array}{c}\text { ROC-AUC analysis } \\
\text { FO vs. F1-4: } \\
\text { Mean: AUC }=\mathbf{0 . 7 8 , 9 5 \%} \text { CI: }[0.72-0.84] \\
F 0-1 \text { vs. } F 2-4: \\
\text { Mean: AUC }=\mathbf{0 . 7 3}[0.67-0.78] \\
F 0-2 \text { vs. } F 3-4: \\
\text { Mean: AUC }=\mathbf{0 . 7 3}[0.67-0.79] \\
F O-3 \text { vs. F4: } \\
\text { Skewness: AUC }=\mathbf{0 . 8 7}[0.83-0.92]\end{array}$ \\
\hline Lubner et al. [13] & Staging of liver fibrosis. & $\begin{array}{l}\text { Single slice of the liver at the } \\
\text { level of the porta hepatis. }\end{array}$ & Histogram-based first-order statistics & - & $\begin{array}{c}\text { ROC-AUC analysis } \\
\text { Multivariable logistic regression: } \\
3 \text { features } \\
\text { F0 vs. F1-4: AUC }=\mathbf{0 . 8 1}[0.78-0.85] \\
\text { F0-1 vs. F2-4: AUC }=\mathbf{0 . 8 1}[0.78-0.85] \\
\text { F0-2 vs. F3-4: AUC }=\mathbf{0 . 8 6}[0.82-0.89] \\
\text { F0-3 vs. F4: AUC }=\mathbf{0 . 8 8}[0.85-0.91]\end{array}$ \\
\hline Kayaalti et al. [10] & Staging of liver fibrosis. & $\begin{array}{c}32 \times 32 \text { pixel ROIs }(n=10 / \\
\text { patient) }\end{array}$ & $\begin{array}{c}\text { First-order statistics, } \\
\text { GLCM features, } \\
\text { Discrete Wavelet Transform, } \\
\text { Discrete Fourier Transform }\end{array}$ & SVM classifier, k-NN classifier & $\begin{array}{c}\text { Pairwise classification accuracy: } \\
4 \text { features } \\
\text { k-NN: } \mathbf{8 6 . 0 7 \%} \\
\text { SVM: } \mathbf{8 9 . 7 0} \%\end{array}$ \\
\hline Kayaalti et al., [8] & Staging of liver fibrosis. & $\begin{array}{l}32 \times 32 \text { pixel ROIs }(n=10 / \\
\text { patient) }\end{array}$ & $\begin{array}{c}\text { First-order statistics, } \\
\text { GLCM, GLRLM, GTDM-based features, } \\
\text { Laws' features, } \\
\text { Discrete Wavelet Transform, } \\
\text { Discrete Fourier Transform, } \\
\text { Gabor filters }\end{array}$ & $\begin{array}{l}\text { SVM classifier, } \\
\text { k-NN classifier }\end{array}$ & $\begin{array}{c}\text { Pairwise classification accuracy: } \\
5 \text { features } \\
\text { k-NN: } \mathbf{9 3 . 1 7 \%} \\
\text { SVM: } \mathbf{9 4 . 0 0 \%} \\
\text { Multi-category classification accuracy: } \\
5 \text { features } \\
\text { k-NN: } \mathbf{4 0 . 5 2} \% \\
\text { SVM: } \mathbf{3 8 . 8 6} \%\end{array}$ \\
\hline Budai et al. [12] & $\begin{array}{l}\text { Differentiating between low- } \\
\text { grade vs. high grade liver } \\
\text { fibrosis. }\end{array}$ & The entire liver volume. & $\begin{array}{c}\text { First-order statistics, } \\
\text { Shape-based features, } \\
\text { GLCM, GLRLM, GLDM, } \\
\text { GLSZM, NGTDM-based features }\end{array}$ & $\begin{array}{l}\text { SVM classifier, } \\
\text { RF classifier }\end{array}$ & $\begin{array}{l}\text { ROC-AUC analysis } \\
\text { Analysis I (equal size training and test sets): } \\
\text { SVM (18 features): AUC }=\mathbf{0 . 7 6}[0.67-0.84] \\
\text { RF (2 features): AUC }=\mathbf{0 . 9 0}[0.85-0.95] \\
\text { Analysis II (against scanner types): } \\
\text { SVM (66 features): AUC }=\mathbf{0 . 9 0}[0.87-0.93] \\
\text { RF (28 features): AUC }=\mathbf{0 . 8 8}[0.84-0.91]\end{array}$ \\
\hline Pickhardt et al. [14] & $\begin{array}{l}\text { Comparing the classification } \\
\text { performance of automated LSN } \\
\text { assessment, radiomic analysis, } \\
\text { volume assessment, and } \\
\text { laboratory parameters in liver } \\
\text { fibrosis staging. }\end{array}$ & $\begin{array}{l}\text { Single slice of the liver at the } \\
\text { level of the porta hepatis. }\end{array}$ & Histogram-based first-order statistics & - & $\begin{array}{c}\text { Multivariable logistic regression analysis: } \\
9 \text { CT-based features }+2 \text { Lab parameters } \\
\text { F0-1 vs. F2-4: } \mathrm{AUC}=\mathbf{0 . 9 2 8} \\
\text { F0-2 vs. F3-4: } \mathrm{AUC}=\mathbf{0 . 9 5 6} \\
\text { F0-3 vs. F4: AUC }=\mathbf{0 . 9 7 2}\end{array}$ \\
\hline
\end{tabular}

GLCM: gray-level co-occurrence matrix, GLGM: gray-level gradient matrix, GLRLM: gray-level run-length matrix, GLDM: gray-level dependence matrix, GLSZM: gray-level size zone matrix, NGTDM: neighboring gray-tone difference matrix, SRHGE: short-run high gray-level emphasis, SVM: support vector machine, RF: random forest, k-NN: k-nearest neighbor, ROC: receiver operating characteristic curve, AUC: area under the receiver operating characteristic curve, CI: confidence interval, ROI: region of interest, LSN: liver surface nodularity 
Table 2. Overview of the literature on the CT texture analysis-based prognosis prediction of patients with hepatocellular carcinoma

\begin{tabular}{|c|c|c|c|c|}
\hline Study & Description & Imaging modality & Texture features & Results \\
\hline Oh et al. [19] & $\begin{array}{l}\text { To identify independent predictors of } \\
\text { DFS of patients with resected HCC. }\end{array}$ & $\begin{array}{l}\text { Arterial phase and portal venous phase } \\
\text { CT scans }\end{array}$ & Histogram-based first-order statistics & $\begin{array}{l}\text { Cox proportional hazards analysis } \\
\text { Arterial phase } \\
\text { Skewness: DFS hazard ratio }=\mathbf{1 0 . 9 6} \\
\text { [95\% CI: } 3.21-37.46]\end{array}$ \\
\hline Defour et al. [20] & $\begin{array}{l}\text { To identify independent predictors of } \\
\text { OS and DFS of patients with resected } \\
\text { HCC. }\end{array}$ & $\begin{array}{l}\text { Arterial phase and portal venous phase } \\
\text { CT scans }\end{array}$ & Histogram-based first-order statistics & $\begin{array}{c}\text { Cox proportional hazards analysis } \\
\text { Arterial phase: OS } \\
\text { Kurtosis: hazard ratio }=\mathbf{3 . 2 3}[1.35- \\
7.71] \\
\text { Portal venous phase: OS } \\
\text { Skewness: hazard ratio }=\mathbf{3 5 3 . 4 4} \\
\text { [1.31-95102.23] } \\
\text { No textural feature was identified as } \\
\text { predictor of DFS. }\end{array}$ \\
\hline Guo et al. [21] & $\begin{array}{l}\text { To assess if radiomic parameters can } \\
\text { predict the DFS of patients with HCC } \\
\text { after liver transplantation. }\end{array}$ & $\begin{array}{l}\text { Arterial phase and portal venous phase } \\
\text { CT scans }\end{array}$ & $\begin{array}{l}\text { Histogram-based first-order statistics, } \\
\text { Shape-based parameters, GLCM, } \\
\text { GLRLM, GLSZM, GLDM, NGTDM- } \\
\text { based features }\end{array}$ & $\begin{array}{c}\text { Cox proportional hazards analysis } \\
8 \text { texture features: DFS } \\
\text { C-index }=\mathbf{0 . 7 0 5}[0.537-0.874]\end{array}$ \\
\hline Park et al. [23] & $\begin{array}{l}\text { To explore the predictive value of } \\
\text { radiomics analysis to select well- } \\
\text { responding patients before TACE. }\end{array}$ & Arterial phase CT scans & $\begin{array}{c}\text { Histogram-based first-order statistics, } \\
\text { Shape-based parameters, GLCM-based } \\
\text { features }\end{array}$ & $\begin{array}{c}\text { ROC-AUC analysis: } \\
\text { Multivariable Logistic Regression } \\
\text { Analysis } \\
\text { GLCM moments: } C R \\
\text { OR }=\mathbf{6 . 5 7}[2.3-23.44] ; \text { AUC }=\mathbf{0 . 6 8} \\
{[0.59-0.77]} \\
\text { Homogeneity: } C R \\
\text { OR }=\mathbf{0 . 6 9}[0.52-0.89] ; \text { AUC }=\mathbf{0 . 7 2} \\
{[0.63-0.80]} \\
\text { Tumor size: } C R \\
\text { OR }=\mathbf{1 7 . 2 6}[3.78-112.77] ; \text { AUC }= \\
\mathbf{0 . 6 2}[0.52-0.72]\end{array}$ \\
\hline Kim et al. [24] & $\begin{array}{l}\text { To build a model for OS prediction in } \\
\text { patients with HCC treated with TACE. }\end{array}$ & Arterial phase CT scans & $\begin{array}{c}\text { Histogram-based first-order statistics, } \\
\text { Shape-based features, GLCM-based } \\
\text { features, intensity size zone matrix- } \\
\text { based features }\end{array}$ & $\begin{array}{c}\text { Cox proportional hazards analysis } \\
5 \text { radiomic features }+3 \text { clinical } \\
\text { parameters: OS } \\
\text { hazard ratio }=\mathbf{1 9 . 8 8}[6.37-62.02]\end{array}$ \\
\hline Mulé et al. [25] & $\begin{array}{c}\text { To investigate the potential of CTTA in } \\
\text { therapeutic decision making by } \\
\text { predicting treatment response in } \\
\text { advanced HCC. }\end{array}$ & $\begin{array}{c}\text { Arterial phase and portal venous phase } \\
\text { CT scans }\end{array}$ & Histogram-based first-order statistics & $\begin{array}{l}\text { Cox proportional hazards analysis } \\
\text { Portal venous phase: OS } \\
\text { Entropy: hazard ratio }=\mathbf{5 . 0 8}[1.72- \\
14.98]\end{array}$ \\
\hline
\end{tabular}


performance than the PV phase or combined signatures. A nomogram compiled from the radiomics signature and clinical risk factors was a good predictor of DFS with a concordance index of 0.785 in the training dataset and 0.789 in the validation dataset.

Trans-arterial chemoembolization (TACE) is a standard treatment to control tumor growth and to prolong survival in patients with non-resectable HCC [22]. The detection of treatment response after TACE is essential for determining the optimal timing of the repeats or the need for switching to alternative ablative or systemic therapies. Multiple publications attempted to explore the predictive value of radiomics analysis to select well-responding patients before TACE (Table 2). Park et al. [23] collected pretreatment CT images of 132 HCCs from 96 patients and subjected them to both $2 \mathrm{D}$ and $3 \mathrm{D}$ texture feature extraction. Tumors with complete response (CR) following TACE showed significantly lower homogeneity, higher mean attenuation, and gray-level cooccurrence matrix (GLCM) moments compared to partial responders. Among all radiomic parameters, higher GLCM moments, lower homogeneity, and smaller tumor size proved to be independent predictors of $\mathrm{CR}$ and time to progression (TTP) in a multivariable analysis. Kim et al. [24] retrospectively investigated the baseline CT scans of 88 HCCs treated with TACE. They constructed a radiomics score model by selecting a linear combination of features with the LASSO Cox regression method that could predict overall survival (OS). The final radiomics model included histogram-based features (median, kurtosis, and energy), shape-based features (spherical disproportion and surfaceto-volume ratio), GLCM-based features (energy, informational measure of correlation, maximum probability, contrast, and sum average), and intensity size zone matrixbased features (size zone variability). The predictive accuracy of the radiomics score model was compared with the clinical score and the combined score models. The combined score model included five radiomic features (surface-areato-volume ratio, kurtosis, median, gray-level co-occurrence matrix contrast, and size zone variability) and three clinical factors (Child-Pugh score, $\alpha$-fetoprotein level, and HCC size). The combined score model was a better predictor of OS (hazard ratio: 19.88; 95\% CI:6.37-62.02; $P<0.0001$ ) than the other two models. Thus, the authors of the above studies were able to convincingly demonstrate that pretreatment radiomics analysis of HCCs could be a useful method for selecting the best-fit candidates for TACE.

Unfortunately, HCC is often diagnosed in an advanced stage, when curative treatment by resection or local ablation is not available. Sorafenib is the first systemic treatment, which has improved the survival of patients with advanced-stage HCC in clinical trials. However, there can be significant differences between patients in terms of survival benefit and adverse reactions. Mulé et al. [25] were among the first to investigate the potential of CTTA in therapeutic decision making by predicting treatment response in advanced HCC. Their study included 92 patients with advanced HCC, who were treated with sorafenib. They performed texture analysis at the arterial and PV phase of pretreatment CT scans and found that the entropy at the PV phase was an independent predictor of OS. Meanwhile, another study authored by Fu et al. [26] explored CTTA's ability to drive patient selection before combination treatment with TACE and sorafenib would take place. Through the analysis of 261 HCCs, out of which 64 were treated with the combination and 197 with TACE only, the authors found that CTTA parameters derived from PV phase images, independent of tumor number or size, are correlated with TTP and OS. Also, patients with lower Gabor-1-90 and wavelet-3-D values were the most likely to benefit from the TACE and sorafenib combination.

Radiomic analysis has achieved promising results in other primary liver neoplasms, such as intrahepatic cholangiocarcinoma (ICC). Sadot et al. [27] correlated radiomic features with immunohistochemistry profiles in 25 ICCs. In their cohort, the expression of hypoxia markers such as vascular endothelial growth factor and epidermal growth factor receptor was associated with quantitative imaging phenotypes, which were likely to represent variations in tumor perfusion. According to a recent publication, which reported the analysis of 52 CT images of cholangiocarcinomas, CT derived texture features showed a significant association with both tumor stage and the time to recurrence [28]. The authors concluded that quantitative texture parameters were promising for the prediction of patient outcomes in ICC. The usefulness of CTTA to identify ICCs, which best respond to trans-arterial radioembolization (TARE) was also explored in a recent publication. Mosconi et al. [29] performed a retrospective texture analysis on pretreatment CT scans of 55 ICC patients. Tumors showing objective response after TARE had higher iodine uptake implicated by the higher mean histogram intensity, and more homogeneous distribution of iodine implicated by the lower kurtosis, GLCM contrast, and GLCM dissimilarity, as well as the higher GLCM correlation and GLCM homogeneity at the baseline CT images. A favorable radiomic signature was detected in 15 patients, and it was associated with prolonged post-TARE progression-free survival.

Radiomics-based machine learning models have also been successful in guiding the differential diagnosis of focal liver lesions. A radiomics nomogram (Nomo-Score), built from texture features and clinical parameters, was able to reliably differentiate between HCC and focal nodular hyperplasia $(\mathrm{FNH})$ at contrast-enhanced CT scans [30]. Nie et al. extracted a radiomics signature (Rad-Score) with LASSO analysis and combined it with clinical factors. The cohort of $55 \mathrm{FNH}$ and $101 \mathrm{HCC}$ cases was divided into training and validation sets and to assess the prediction accuracy of the three models (clinical factors model, radiomics signature model, combined radiomics nomogram model). The Nomo-Score showed excellent discriminatory ability in the validation set with AUC 0.917 [95\% CI:0.800$1.000]$, respectively and outperformed both the clinical factors and the radiomics signature models in the classification of HCC vs. FNH (AUC = 0.769; 95\% CI:0.590-0.949 and AUC $=0.865 ; 95 \%$ CI:0.725-1.000): The radiomics nomogram also demonstrated a higher overall net benefit in a decision curve analysis [30]. 


\section{Radiomics analysis of secondary liver tumors}

Secondary liver tumors are the most frequent liver malignancies. In colorectal cancer (CRC), liver metastases can be detected in approximately $20 \%$ of the patients at the time of the diagnosis, and up to $50 \%$ of patients develop metastases overall [31]. Miles et al. [32] were among the first to investigate the potential of CTTA for predicting the prognosis of colorectal liver metastases (CRLM). The uniformity of the liver on PV phase images with 10- and 12-pixels filter size showed the least variation, and it also was the best predictor of survival in the study cohort comprising of 48 patients. Meanwhile, the authors did not find a significant correlation between survival and hepatic perfusion parameters.

CTTA may also improve the clinical management of CRLMs by facilitating early detection and guiding therapeutic decision making. Surgical resection is the most effective treatment of CRLM, as it results in the best longterm survival [31]. The early detection of CRLMs is essential to preserve the chance of curative resection. Occult liver metastases are often detected during the histopathological examination of the resected liver, and they are the presumed origin of metachronous liver metastases in CRC patients. CTTA may hold promise to detect CRLMs at an early stage that is not recognizable through visual inspection. Rao et al. [33] collected PV phase CT images of 29 patients with either synchronous $(n=10)$, or metachronous $(n=4)$, or with no liver metastases $(n=15)$; and they performed CTTA of the visibly non-affected liver parenchyma. The entropy of the lesion free parenchyma was significantly higher, and the uniformity was significantly lower in the group with synchronous liver metastasis compared with the non-metastatic group. The receiver operating characteristics (ROC) curve analysis showed 0.78 [95\% CI:0.57-0.99] AUC for the diagnosis of synchronous metastases based on the selected texture parameters. However, there was no difference in texture parameters between the group with metachronous metastasis and the other groups.

Taghavi et al. [34] also evaluated the ability of a radiomics-based machine learning prediction model to forecast the occurrence of metachronous CRLMs. They calculated radiomic features of the liver parenchyma at $\mathrm{PV}$ phase CT scans of 91 CRC patients, out of who 24 patients did and 67 patients did not develop metachronous CRLMs during the 24-month follow-up. A random forest (RF) model constructed from radiomic features outperformed the model based on clinical features and showed similar performance as the combined model for the prediction of metachronous metastases in both the training and the validation groups.

Systemic chemotherapy is routinely used for the treatment of surgically non-resectable tumors, or to reduce CRLMs to a surgically resectable size. The response evaluation criteria in solid tumors (RECIST 1.1) system is typically applied for the objective assessment of CRLMs response to systemic therapy with imaging studies. However, RECIST 1.1 mostly relies on the changes in cross-sectional diameters and does not account for spatial heterogeneity inside the tumors. Rao et al. used CTTA to predict the treatment response to systemic chemotherapy in 21 CRLM using histology grading as a reference. The best-performing texture parameters, uniformity and entropy, had stronger predictive values in a multivariable logistic regression analysis than conventional image markers, such as tumor size and volume [35].

The PRODIGE-9 multicenter prospective study recruited 491 patients treated with 5-fluorouracil, leucovorin, irinotecan (FOLFIRI), and bevacizumab for CRLMs. As an ancillary study from PRODIGE-9, Dohan et al. built a radiomics nomogram [Survival PrEdiction in patients treated by FOLFIRI and bevacizumab for metastatic CRC using contrast-enhanced CT TextuRe Analysis (SPECTRA) Score] to estimate OS based on PV phase CT images taken at baseline and two months after the initiation of treatment. Three radiomic features: the sum of target lesions, the baseline density of the dominant lesion, and the absolute change in kurtosis with a medium spatial scale filter size were included in the final SPECTRA Score based on a multivariable Cox analysis of data from 120 patients in the training cohort. Patients with scores above the median $(>0.02)$ had significantly lower OS in the training cohort and in both a validation cohort of 110 patients and an external validation cohort of 40 patients. The SPECTRA Score calculated at two months was able to estimate OS with the same accuracy as RECIST1.1 at six months in both the training and the validation cohorts [36].

The feasibility of radiomic analysis to predict the treatment response of liver metastasis of esophagogastric cancer was assessed by Klaassen et al. [37] in 196 lesions of 18 patients treated with chemotherapy. They constructed multiple RF models using features derived from contrastenhanced pretreatment CT scans and divided patients into groups of non-responders, partial responders (PR), and complete remission (CR) based on a follow-up CT. The trained model resulted in an average 0.65 [95\% CI:0.570.73 ] and 0.79 [95\% CI:0.72-0.87] AUC for the PR and CR groups, respectively, in the validation set.

\section{Exploration of liver lesions with radiogenomics}

Rapid advancements in the genetic and genomic profiling of abdominal malignancies during the past decades have transformed the diagnostics of oncology patients and made personalized cancer therapy available. In the future, state-ofthe-art imaging modalities can complete a detailed characterization of a tumor's phenotype by depicting morphology and metabolic activity. Radiogenomics is an emerging new field of medical imaging where imaging biomarkers are correlated with molecular and genetic subtypes of pathologic lesions (Table 3). It has the potential to improve the noninvasive classification and surveillance of neoplasias, and may also contribute to patient-specific therapeutic decision making [38].

In a ground-breaking study, Segal et al. [39] were among the first to correlate the genomics of tumors with imaging features in the clinical setting. The authors extracted 32 
Table 3. Overview of the literature on Radiogenomics of focal liver lesions

\begin{tabular}{|c|c|c|c|c|}
\hline Study & Description & Imaging modality & Texture features & Results \\
\hline Segal et al. [39] & $\begin{array}{l}\text { To correlate the genomics of HCCs with } \\
\text { imaging traits. }\end{array}$ & $\begin{array}{c}\text { Unenhanced, arterial, and portal venous } \\
\text { phase CT scans }\end{array}$ & - & $\begin{array}{l}\text { The combinations of only } 9 \text { imaging } \\
\text { traits could explain } \mathbf{5 0 \%} \text { of the entire } \\
\text { transcriptome }\end{array}$ \\
\hline Kuo et al. [40] & $\begin{array}{c}\text { To correlate the genomics of HCCs with } \\
\text { imaging traits. }\end{array}$ & $\begin{array}{l}\text { Unenhanced, arterial, and portal venous } \\
\text { phase CT scans }\end{array}$ & - & $\begin{array}{c}\text { Tumor margin score on arterial phase } \\
\text { images was significantly associated with } \\
\text { a predefined gene expression signature } \\
\text { of doxorubicin response }(\boldsymbol{P}=\mathbf{0 . 0 0 5 6}), \\
\text { venous invasion }(\boldsymbol{P}=\mathbf{0 . 0 0 4 7}) \text {, and } \\
\text { tumor stage }(\boldsymbol{P}=\mathbf{0 . 0 0 1 9}) .\end{array}$ \\
\hline Banerjee et al. [41] & $\begin{array}{l}\text { To correlate the RVI score with MVI in } \\
\text { patients with HCC. }\end{array}$ & $\begin{array}{l}\text { Arterial phase and portal venous phase } \\
\text { CT scans }\end{array}$ & - & $\begin{array}{c}\text { Diagnostic accuracy in predicting MVI: } \\
\mathbf{8 9 \%} \\
\text { Positive RVI associated with lower OS } \\
(\boldsymbol{P}<\mathbf{0 . 0 0 1}) \text { and lower RFS }(\boldsymbol{P}=\mathbf{0 . 0 0 1})\end{array}$ \\
\hline West et al. [42] & $\begin{array}{l}\text { To correlate imaging features with the } \\
\text { expression levels of genes associated } \\
\text { with doxorubicin resistance in HCCs. }\end{array}$ & $\begin{array}{c}\text { Baseline CT scans from The Cancer } \\
\text { Imaging Archive }\end{array}$ & $\begin{array}{l}\text { First-order statistics, GLCM-based } \\
\text { features }\end{array}$ & $\begin{array}{c}\text { ROC-AUC analysis } \\
\text { TP53 prediction: } \text { AUC }=\mathbf{0 . 8 6} \\
\text { TOP2A prediction: } \text { AUC }=\mathbf{0 . 7 8} \\
\text { CTNNB1 prediction: } \text { AUC }=\mathbf{0 . 8 6} \\
\text { CDKN2A prediction: } \text { AUC }=\mathbf{0 . 7 6} \\
\text { AKT1 prediction: } \text { AUC }=\mathbf{0 . 7 2}\end{array}$ \\
\hline Lubner et al. [44] & $\begin{array}{c}\text { To correlate radiomic features with } \\
\text { histology grade, baseline CEA and } \\
\text { KRAS mutation status. }\end{array}$ & Portal venous phase CT scans & Histogram-based first-order statistics & $\begin{array}{c}\text { No significant association was seen } \\
\text { between baseline serum CEA and } \\
\text { texture features. } \\
\text { Entropy }(\boldsymbol{P}=\mathbf{0 . 0 0 7}), \text { MPP ( } \boldsymbol{P}= \\
\mathbf{0 . 0 0 2}) \text {, and SD of the pixel histogram } \\
(\boldsymbol{P}=\mathbf{0 . 0 0 4}) \text { showed negative } \\
\text { association with tumor grade. } \\
\text { Skewness showed inverse association } \\
\text { with KRAS mutation with OR }=\mathbf{0 . 1 5} \text {, } \\
\boldsymbol{P}=\mathbf{0 . 0 4} .\end{array}$ \\
\hline
\end{tabular}

HCC: hepatocellular carcinoma, MVI: microvascular invasion, RVI: radiogenomic venous invasion, RFS: recurrence-free survival, OS: overall survival, GLCM: gray-level co-occurrence matrix, ROC: receiver operating characteristic curve, AUC: area under the receiver operating characteristic curve, CEA: carcinoembryonic antigen, MPP: mean of positive pixels, SD: standard deviation, OR: odds ratio 
distinctive imaging traits from three-phase contrastenhanced CT images of 28 HCCs. The genome-wide expression profiles of the tumors encompassing 6,732 genes were detected with microarray analysis. The authors performed a module network analysis to construct an association map consisting of 116 gene expression modules, all of which were associated with specific combinations of imaging traits that could explain the aggregate expression level within the module. The combinations of 28 imaging traits were sufficient to determine all of the gene expression modules, and the combinations of only nine imaging traits could explain $50 \%$ of the entire transcriptome. When the model was applied to an independent validation set of 19 HCCs, imaging traits derived from pre-operative CT scans were able to correctly predict expression levels in 71 out of the 116 genomic modules. The modules often contained genes that shared common biological or regulatory functions, such as cell proliferation, extracellular matrix remodeling, or synthesis of liver-specific enzymes.

Kuo et al. [40] analyzed six imaging phenotypes in the CT scans of 30 HCC patients. The association between imaging scores and genomic data, including 18,000 tumorderived gene expression profiles, was assessed with both supervised and unsupervised analysis. The imaging trait, which characterized tumor margins on arterial phase images, was significantly associated with a predefined gene expression signature of doxorubicin response, venous invasion, and tumor stage.

Banerjee et al. [41] used the genomic data of 157 HCC patients to construct an association map, which could determine the correlation between a gene expression signature of venous invasion and imaging traits of histologically proven microvascular invasion (MVI). They created a radiogenomic venous invasion (RVI) score from a combination of three imaging traits, which showed substantial interobserver agreement and could predict MVI on contrast-enhanced CT with 89\%, 76\%, and 94\%, accuracy, sensitivity, and specificity, respectively. Patients with RVI positive tumors had a significantly lower OS and recurrence-free survival rate at three years compared to RVInegative cases.

A study by West et al. [42] retrospectively analyzed CT images and gene expression profiles of 27 treatment-naïve HCC patients from The Cancer Imaging Archive and The Cancer Genome Atlas. The texture parameters, which best described the heterogeneity of tumors, were correlated with the expression levels of five genes known to be associated with doxorubicin chemoresistance. The radiomic features were able to identify TP53 status with AUC $=0.87$, specificity $=92.31 \%$, and sensitivity $=92.9 \%$, and CTNNB1 status with $\mathrm{AUC}=0.87$, specificity $=92.3 \%$, and sensitivity $=85.7 \%$. The authors concluded that doxorubicin chemoresistance could be reliably predicted by the radiogenomic analysis of pretreatment CT scans.

In a study by DeLeon et al. [43], CTTA features were analyzed in 33 ICCs, 12 of which were positive for fibroblast growth factor receptor 2 (FGFR2) fusion. FGFR2 fusion is a frequent genomic alteration in ICC, which is also a predictor of better prognosis and response to treatment with tyrosine kinase inhibitors. A prediction model constructed from radiomic features achieved $90 \%$ accuracy, sensitivity $=87 \%$, and specificity $=94 \%$ during the classification of FGFR2 fusion. The results demonstrated that CTTA combined with machine learning might have the potential to detect genetic aberrations in ICCs. Lubner et al. [44] analyzed single CRLMs on pretreatment portal phase CT images of 77 patients. Texture parameters were correlated with histology grade, baseline carcinoembryogenic antigen (CEA), and KRAS mutation status. The authors found that skewness was negatively associated with KRAS mutations, while entropy at coarse filtration level was a significant predictor of OS.

\section{Conclusion}

Our Review indicates that machine-learning-based CT texture analysis has the potential to differentiate between benign and malignant liver lesions, and as previous studies have also proved it, CTTA can predict the stages of liver fibrosis non-invasively. With further advancement and improvement of technology within the field of radiomics, it may one day serve as a fully automated non-invasive assessment tool to assist and guide clinicians in evaluating the prognosis for individual patients. Meanwhile, radiogenomics has the potential to detect tumor-specific gene expression profiles and to substitute for the detailed characterization of molecular and genetic subtypes in a set of liver pathologies. It may help to obtain an earlier and more precise diagnosis compared to conventional imaging and may guide patient-specific treatments. However, as it is a relatively new field in radiology, further research is necessary before radiomics can be introduced into standard patient care.

Authors' contributions: BKB - Conceptualization, Data curation, Drafting of the manuscript, Preparing the Figures.

VF - Conceptualization, Drafting of the manuscript, Proofreading.

SS - Conceptualization, Drafting of the manuscript, Proofreading.

BF - Data curation, Drafting of the manuscript.

AT - Data curation, Drafting of the manuscript.

VO - Data curation, Drafting of the manuscript.

VB - Drafting of the manuscript, Critical revision.

PNK - Conceptualization, Drafting of the manuscript, Critical revision.

All authors reviewed the final version of the manuscript and agreed to submit it to IMAGING for publication.

Funding sources: PNK (Bolyai 386/2017) was supported by the János Bolyai Research Scholarship of the Hungarian Academy of Sciences (https://mta.hu/bolyai-osztondij). $\mathrm{BKB}, \mathrm{AT}, \mathrm{VB}$ and PNK received financial support from the GINOP 2-2-18 grant. The funders had no role in the study design, data collection, and analysis, decision to publish, or preparation of the manuscript. 
Conflict of interest: The authors have no conflict of interest to disclose.

\section{ACKNOWLEDGEMENTS}

Not applicable.

\section{REFERENCES}

[1] Sutton RN, Hall EL: Texture measures for automatic classification of pulmonary disease. IEEE Trans Comput 1972; C-21(7): 667-76.

[2] Lambin P, Rios-Velazquez E, Leijenaar R, Carvalho S, van Stiphout RG, Granton P, et al.: Radiomics: extracting more information from medical images using advanced feature analysis. Eur J Cancer 2012; 48(4): 441-6.

[3] Zwanenburg A, Leger S, Vallières M, Löck S: Image biomarker standardisation initiative. arXiv preprint arXiv:1612.07003 2016.

[4] Kumar V, Gu Y, Basu S, Berglund A, Eschrich SA, Schabath MB, et al.: Radiomics: the process and the challenges. Magn Reson Imaging 2012; 30(9): 1234-48.

[5] Naganawa S, Enooku K, Tateishi R, Akai H, Yasaka K, Shibahara J, et al.: Imaging prediction of nonalcoholic steatohepatitis using computed tomography texture analysis. Eur Radiol 2018; 28(7): 3050-8.

[6] Daginawala N, Li B, Buch K, Yu H, Tischler B, Qureshi MM, et al.: Using texture analyses of contrast enhanced CT to assess hepatic fibrosis. Eur J Radiol 2016; 85(3): 511-7.

[7] Lubner MG, Malecki K, Kloke J, Ganeshan B, Pickhardt PJ: Texture analysis of the liver at MDCT for assessing hepatic fibrosis. Abdom Radiol (NY) 2017; 42(8): 2069-78.

[8] Kayaaltı Ö, Aksebzeci BH, Karahan İÖ, Deniz K, Öztürk M, Yllmaz B, et al.: Liver fibrosis staging using CT image texture analysis and soft computing. Appl Soft Comput 2014; 25: 399-413.

[9] Zhang X, Gao X, Liu BJ, Ma K, Yan W, Liling L, et al.: Effective staging of fibrosis by the selected texture features of liver: which one is better, CT or MR imaging? Comput Med Imaging Graph 2015; 46(Pt 2): 227-36.

[10] Kayaaltı Ö, Aksebzeci BH, Karahan IÖ, Deniz K, Öztürk M, Yilmaz B, et al., editors. Staging of the liver fibrosis from CT images using texture features. 2012 7th International Symposium on Health Informatics and Bioinformatics; 2012 19-22 April 2012.

[11] Kaposi PN, Budai B, Frank V, Shariati S, Folhoffer A, Abonyi M, et al.: CT texture analysis in liver fibrosis shows a strong correlation with portal phase enhancement and can predict cirrhosis in decision tree model. Insights Imaging 2019; 10.

[12] Budai BK, Toth A, Borsos P, Frank VG, Shariati S, Fejer B, et al.: Three-dimensional CT texture analysis of anatomic liver segments can differentiate between low-grade and high-grade fibrosis. BMC Med Imaging 2020; 20(1): 108.

[13] Lubner MG, Jones D, Kloke J, Said A, Pickhardt PJ: CT texture analysis of the liver for assessing hepatic fibrosis in patients with hepatitis C virus. Br J Radiol 2019; 92(1093): 20180153.

[14] Pickhardt PJ, Graffy PM, Said A, Jones D, Welsh B, Zea R, et al.: Multiparametric CT for noninvasive staging of hepatitis $\mathrm{C}$ virus- related liver fibrosis: correlation with the histopathologic fibrosis score. AJR Am J Roentgenol 2019; 212(3): 547-53.

[15] Perrin T, Midya A, Yamashita R, Chakraborty J, Saidon T, Jarnagin WR, et al.: Short-term reproducibility of radiomic features in liver parenchyma and liver malignancies on contrast-enhanced CT imaging. Abdom Radiol 2018; 43(12): 3271-8.

[16] Sayiner M, Golabi P, Younossi ZM: Disease burden of hepatocellular carcinoma: a global perspective. Dig Dis Sci 2019; 64(4): 910-7.

[17] Forner A, Reig M, Bruix J: Hepatocellular carcinoma. Lancet 2018; 391(10127): 1301-14.

[18] Mazzaferro V, Bhoori S, Sposito C, Bongini M, Langer M, Miceli $\mathrm{R}$, et al.: Milan criteria in liver transplantation for hepatocellular carcinoma: an evidence-based analysis of 15 years of experience. Liver Transpl 2011; 17(S2): S44-57.

[19] Oh J, Lee JM, Park J, Joo I, Yoon JH, Lee DH, et al.: Hepatocellular carcinoma: texture analysis of preoperative computed tomography images can provide markers of tumor grade and disease-free survival. Korean J Radiol 2019; 20(4): 569-79.

[20] Brenet Defour L, Mulé S, Tenenhaus A, Piardi T, Sommacale D, Hoeffel C, et al.: Hepatocellular carcinoma: CT texture analysis as a predictor of survival after surgical resection. Eur Radiol 2019; 29(3): 1231-9.

[21] Guo D, Gu D, Wang H, Wei J, Wang Z, Hao X, et al.: Radiomics analysis enables recurrence prediction for hepatocellular carcinoma after liver transplantation. Eur J Radiol 2019; 117: 33-40.

[22] Heimbach JK, Kulik LM, Finn RS, Sirlin CB, Abecassis MM, Roberts LR, et al.: AASLD guidelines for the treatment of hepatocellular carcinoma. Hepatology 2018; 67(1): 358-80.

[23] Park HJ, Kim JH, Choi S-y, Lee ES, Park SJ, Byun JY, et al.: Prediction of therapeutic response of hepatocellular carcinoma to transcatheter arterial chemoembolization based on pretherapeutic dynamic CT and textural findings. AJR Am J Roentgenol 2017; 209(4): W211-20.

[24] Kim J, Choi SJ, Lee S-H, Lee HY, Park H: Predicting survival using pretreatment CT for patients with hepatocellular carcinoma treated with transarterial chemoembolization: comparison of models using radiomics. AJR Am J Roentgenol 2018; 211(5): 1026-34.

[25] Mulé S, Thiefin G, Costentin C, Durot C, Rahmouni A, Luciani A, et al.: Advanced hepatocellular carcinoma: pretreatment contrastenhanced CT texture parameters as predictive biomarkers of survival in patients treated with sorafenib. Radiology 2018; 288(2): 445-55.

[26] Fu S, Chen S, Liang C, Liu Z, Zhu Y, Li Y, et al.: Texture analysis of intermediate-advanced hepatocellular carcinoma: prognosis and patients' selection of transcatheter arterial chemoembolization and sorafenib. Oncotarget 2017; 8(23): 37855-65.

[27] Sadot E, Simpson AL, Do RKG, Gonen M, Shia J, Allen PJ, et al.: Cholangiocarcinoma: correlation between molecular profiling and imaging phenotypes. PLoS ONE 2015; 10(7): e0132953.

[28] King MJ, Hectors S, Lee KM, Omidele O, Babb JS, Schwartz M, et al.: Outcomes assessment in intrahepatic cholangiocarcinoma using qualitative and quantitative imaging features. Cancer Imaging 2020; 20(1): 43.

[29] Mosconi C, Cucchetti A, Bruno A, Cappelli A, Bargellini I, De Benedittis $C$, et al.: Radiomics of cholangiocarcinoma on pretreatment CT can identify patients who would best respond to radioembolisation. Eur Radiol 2020; 30(8): 4534-44. 
[30] Nie P, Yang G, Guo J, Chen J, Li X, Ji Q, et al.: A CT-based radiomics nomogram for differentiation of focal nodular hyperplasia from hepatocellular carcinoma in the non-cirrhotic liver. Cancer Imaging 2020; 20(1): 20.

[31] Van Cutsem E, Cervantes A, Nordlinger B, Arnold D: Metastatic colorectal cancer: ESMO Clinical Practice Guidelines for diagnosis, treatment and follow-up. Ann Oncol 2014; 25: iii1-9.

[32] Miles KA, Ganeshan B, Griffiths MR, Young RCD, Chatwin CR: Colorectal cancer: texture analysis of portal phase hepatic CT images as a potential marker of survival. Radiology 2009; 250(2): $444-52$.

[33] Rao S-X, Lambregts DMJ, Schnerr RS, van Ommen W, van Nijnatten TJA, Martens MH, et al.: Whole-liver CT texture analysis in colorectal cancer: does the presence of liver metastases affect the texture of the remaining liver? United Eur Gastroenterol J 2014; 2(6): $530-8$.

[34] Taghavi, M, Trebeschi, S, Simões, R, Meek, D, Beckers, R, Lambregts, D, et al.: Machine learning-based analysis of CT radiomics model for prediction of colorectal metachronous liver metastases. Abdom Radiol 2021; 46(1): 249-56.

[35] Rao S-X, Lambregts DMJ, Schnerr RS, Beckers RCJ, Maas M, Albarello F, et al.: CT texture analysis in colorectal liver metastases: a better way than size and volume measurements to assess response to chemotherapy? United Eur Gastroenterol J 2015; 4(2): 257-63.

[36] Dohan A, Gallix B, Guiu B, Le Malicot K, Reinhold C, Soyer P, et al.: Early evaluation using a radiomic signature of unresectable hepatic metastases to predict outcome in patients with colorectal cancer treated with FOLFIRI and bevacizumab. Gut 2020; 69(3): 531 .
[37] Klaassen R, Larue RTHM, Mearadji B, van der Woude SO, Stoker J, Lambin $\mathrm{P}$, et al.: Feasibility of $\mathrm{CT}$ radiomics to predict treatment response of individual liver metastases in esophagogastric cancer patients. PLoS One 2018; 13(11): e0207362.

[38] Lo Gullo R, Daimiel I, Morris EA, Pinker K: Combining molecular and imaging metrics in cancer: radiogenomics. Insights Imaging 2020; 11(1): 1 .

[39] Segal E, Sirlin CB, Ooi C, Adler AS, Gollub J, Chen X, et al.: Decoding global gene expression programs in liver cancer by noninvasive imaging. Nat Biotechnol 2007; 25(6): 675-80.

[40] Kuo MD, Gollub J, Sirlin CB, Ooi C, Chen X: Radiogenomic analysis to identify imaging phenotypes associated with drug response gene expression programs in hepatocellular carcinoma. J Vasc Interv Radiol 2007; 18(7): 821-30.

[41] Banerjee S, Wang DS, Kim HJ, Sirlin CB, Chan MG, Korn RL, et al.: A computed tomography radiogenomic biomarker predicts microvascular invasion and clinical outcomes in hepatocellular carcinoma. Hepatology 2015; 62(3): 792-800.

[42] West DL, Kotrotsou A, Niekamp AS, Idris T, Giniebra Camejo D, Mazal NJ, et al.: CT-based radiomic analysis of hepatocellular carcinoma patients to predict key genomic information. J Clin Oncol 2017; 35(15_suppl): e15623-e15623.

[43] DeLeon T, Borad MJ, Wu T, Li J, Zwart CM, Yang G, et al.: Utility of CT texture for identification of FGFR2 fusion in cholangiocarcinoma: a radiogenomic pilot study. J Clin Oncol 2017; 35(15_suppl): e15626-e15626.

[44] Lubner MG, Stabo N, Lubner SJ, del Rio AM, Song C, Halberg RB, et al.: CT textural analysis of hepatic metastatic colorectal cancer: pre-treatment tumor heterogeneity correlates with pathology and clinical outcomes. Abdom Imaging 2015; 40(7): 2331-7. 